\title{
Extremely fast gas/liquid reactions in flow microreactors: carboxylation of short-lived organolithiums.
}

$\operatorname{AUTHOR}(\mathrm{S})$ :

Nagaki, Aiichiro; Takahashi, Yusuke; Yoshida, Junichi

\section{CITATION:}

Nagaki, Aiichiro ...[et al]. Extremely fast gas/liquid reactions in flow microreactors: carboxylation of short-lived organolithiums.. Chemistry - A European Journal 2014, 20(26): 7931-7934

\section{ISSUE DATE:}

2014-06-23

URL:

http://hdl.handle.net/2433/199603

\section{RIGHT:}

This is the peer reviewed version of the following article: Nagaki, A. Takahashi, Y. and Yoshida, J.-i. (2014), Extremely Fast Gas/Liquid Reactions in Flow Microreactors: Carboxylation of Short-Lived Organolithiums. Chem. Eur. J., 20: 7931-7934, which has been published in final form athttp://dx.doi.org/10.1002/chem.201402520. This article may be used for non-commercial purposes in accordance with Wiley Terms and Conditions for Self-Archiving.; この論文は出版 社版でありません。引用の際には出版社版をご確認ざ利用ください。； This is not the published version. Please cite only the published version. 


\title{
Extremely Fast Gas/Liquid Reactions in Flow Microreactors:
}

\section{Carboxylation of Short-Lived Organolithiums}

\author{
Aiichiro Nagaki, ${ }^{[a]}$ Yusuke Takahashi, ${ }^{[a]}$ and Jun-ichi Yoshida ${ }^{[a] *}$
}

\author{
[a] Dr. A. Nagaki, Y. Takahashi, and Prof. J. Yoshida
}

Department of Synthetic and Biological Chemistry, Graduate School of Engineering, Kyoto University Nishikyo-ku, Kyoto, 6158510 (Japan)

Fax: (+81)75-383-2727

E-mail: yoshida@sbchem.kyoto-u.ac.jp

http://www.sbchem.kyoto-u.ac.jp/yoshida-lab/index_e.html

Supporting information for this article is available on the WWW under http://www.chemeurj.org/ or from the author.

Carboxylic acids and their derivatives are important classes of compounds in organic chemistry. Although various methods for synthesis of carboxylic acids have been used so far, carboxylation using $\mathrm{CO}_{2}$ gas as a carbon feedstock ${ }^{[1]}$ is useful and attractive from an environmental point of view. ${ }^{[2]}$ Despite recent remarkable advances in this field, ${ }^{[3]}$ classical carboxylation of organometallics with $\mathrm{CO}_{2}$ still serves as a powerful method because of efficiency and convenience. ${ }^{[4]}$ However, the method suffers from the problem of incompatibility of electrophilic functional groups such as nitro, cyano, and carbonyl groups, which causes severe limitations in the synthesis of carboxylic acids and their derivatives having a variety of functions and biological activities.

Recently, we have reported that various short-lived highly unstable organolithiums such as aryllithiums bearing electrophilic functional groups could be generated and used for reactions with subsequently added electrophiles before they decompose ${ }^{[5]}$ by taking advantages extremely short residence times, which is one of the characteristic features of flow microreactors. ${ }^{[6-8]}$

There are two hurdles that we expected in the implementation of $\mathrm{CO}_{2}$ as an electrophile in the reactions of short-lived organolithium species; the mass transfer between the $\mathrm{CO}_{2}$ gas phase and the solution phase and the rate of the chemical reaction of organolithium species with dissolved $\mathrm{CO}_{2}$. The first one seems to be mainly the issue of fluidics although the mass transfer rate also depends on the nature of the gas and that of the solution. It is well known that the use of flow microreactors enables fast mass transfer in gas-liquid biphasic reactions. ${ }^{[9,10]}$ The second one is the issue of chemistry. The reaction with $\mathrm{CO}_{2}$ should be faster than that with an electrophilic functional group in the organolithium species, although the rate depends on the concentration of $\mathrm{CO}_{2}$ in the solution. The question is what kind of electrophilic functional groups are compatible with the carboxylation. Here, we show that these hurdles were overcome using the flow microreactor system and that aromatic carboxylic acids bearing electrophilic functional groups were synthesized from the corresponding aromatic halides.

The reactions were carried out using a flow microreactor system composed of three T-shaped micromixers (M1, M2 and M3) and three microtube reactors (R1, R2 and R3) as shown in Figure 1. An aryl halide and $n \mathrm{BuLi}$ or PhLi were mixed in M1 and the halogen/lithium exchange was carried out in $\mathbf{R} 1$ to generate the corresponding aryllithium. In the next step, $\mathrm{CO}_{2}$ gas (1.5 eq) that was pressurized to 3.0 bar with a gas pressure regulator valve was introduced at $\mathbf{M} 2$ using a flow controller, and the carboxylation was carried out in R2. In the last step the reaction was quenched by $\mathrm{MeOH}$ at $\mathbf{M} 3$ and $\mathbf{R} 3$.

\section{(Insert Figure 1)}

The carboxylation of $p$-nitrophenyllithium ${ }^{[11]}$ was first examined. The iodine/lithium exchange reaction was carried out with the residence time of $0.014 \mathrm{~s}$ at $-20^{\circ} \mathrm{C}$. In the previous work, ${ }^{[11]}$ we have already revealed that $p$-nitrophenyllithium can be effectively generated under these conditions. We have also revealed that $p$-nitrophenyllithium decomposes with the residence time longer than ca. $0.4 \mathrm{~s}$ at $-20{ }^{\circ} \mathrm{C}$. As shown in Table 1, the corresponding carboxylic acid was obtained in 78\% yield. This means gas/liquid mass transfer and the chemical reaction of $p$-nitrophenyllithium with $\mathrm{CO}_{2}$ are much faster than its decomposition. Under similar conditions $m$ - and $o$-nitrophenyllithiums were carboxylated effectively. The carboxylation reactions of $p$-, $m$-, and $o$-cyanophenyllithiums ${ }^{[12]}$ were also successfully accomplished using the flow microreactor system. The carboxylation of alkoxycarbonylphenyllithiums ${ }^{[13]}$ was more challenging, because esters react with organolithiums very quickly. We have already revealed that $p$-ethoxycarbonylphenyllithium decomposes with the residence time longer than ca. $0.4 \mathrm{~s}$ at $-60{ }^{\circ} \mathrm{C} \cdot{ }^{[13]}$ However, the carboxylation of $p$-ethoxycarbonylphenyllithium could be accomplished to obtain the corresponding carboxylic acid in a reasonable yield. Therefore, the gas/liquid mass transfer and the carboxylation reactions are unexpectedly fast, and are much faster than the decomposition even at low temperatures such as $-60{ }^{\circ} \mathrm{C}$. Thus, carboxylation of aryllithiums bearing electrophilic functional groups, which is very difficult or impossible by conventional batch reactions, was achieved by using flow microreactors.

The carboxylation of phenyllithiums bearing electron-donating substituents such methoxy group and unsubstituted phenyllithium are easier as shown in Table 1 . Notably, the carboxylation of phenyllithium using $\mathrm{CO}_{2}(1.5 \mathrm{eq})^{[14]}$ could be carried out at $0{ }^{\circ} \mathrm{C}$. It is known that at such temperatures the carboxylation of organolithiums using $\mathrm{CO}_{2}$ often leads to the formation of significant amounts of byproducts For example, the carboxylation of phenyllithium at $0{ }^{\circ} \mathrm{C}$ in a batch macro reactor gave a mixture of benzoic acid, benzophenone, and 
triphenylmethanol, although the reaction at $-78{ }^{\circ} \mathrm{C}$ gave benzoic acid in good selectivity (87\% yield) (Scheme 1 ). In contrast, the reaction in the flow microreactor system gave benzoic acid in $87 \%$ yield even at $0{ }^{\circ} \mathrm{C}$. This feature of flow microreactor systems, presumably because of fast heat transfer, is especially important from a view point of industrial production.

\section{(Insert Scheme 1)}

\section{(Insert Table 1)}

The initial products before quenching with methanol in the present reaction are lithium salts of carboxylic acids, which are highly reactive toward activating agents such as TSTU $(O$ - $(N$-succinimidyl $)-1,1,3,3$-tetramethyl uronium tetrafluoroborate $)$ or PyBop $(1 \mathrm{H}$ benzotriazole-1-yloxytris(pyrrolidine-1-yl)phosphonium hexafluorophosphate). Therefore, active esters (esters with a good leaving group), which are commonly used for making peptides from carboxylic acids can be directly synthesized. Thus, the product solutions were treated with the activating agents without methanol quenching. No intentionally added base was required. The corresponding active esters were obtained in good yields as shown in Figure 2.

\section{(Insert Figure 2)}

$\mathrm{N}$-succinimidyl-4- $\left[{ }^{18} \mathrm{~F}\right]$ fluorobenzoate $\left(\left[{ }^{18} \mathrm{~F}\right] \mathrm{SFB}\right)$ is most commonly used to acylate lysine residues and $\mathrm{N}$-terminal amine groups of peptides for positron emission tomography (PET). ${ }^{[15]}$ The following application to this protocol demonstrates the power of the present method, although cold ${ }^{19} \mathrm{~F}$ was used instead of hot ${ }^{18} \mathrm{~F}$. The carboxylation of $p$-fluorophenyllithium generated from 1-bromo-4fluorobenzene with $\mathrm{CO}_{2}$ followed by the reaction with TSTU was completed within 1 min to give the $N$-succinimidyl-4-fluorobenzoate (SFB) in 55\% yield. Then, a solution of SFB and triethylamine in $\mathrm{CH}_{3} \mathrm{CN}$ was added to a solution of cyclo(Arg-Gly-Asp-D-Phe-Lys) (c(RGDfK)) in $\mathrm{H}_{2} \mathrm{O}$ and $\mathrm{CH}_{3} \mathrm{CN}$. The mixture was incubated at $37^{\circ} \mathrm{C}$ for $10 \mathrm{~min}$ and was purified by preparative HPLC to give the desired coupling product in $91 \%$ (Figure 3). The present fast synthesis also demonstrates the possibility of synthesizing compounds containing short-lived positron emitting radionuclide ${ }^{11} \mathrm{C}\left(\mathrm{t}_{1 / 2}=20.4 \mathrm{~min}\right)$ using ${ }^{11} \mathrm{CO}_{2}{ }^{[16]}$.

\section{(Insert Figure 3)}

In conclusion, we have developed an extremely fast and efficient method for carboxylation of short-lived organolithiums with $\mathrm{CO}_{2}$ gas by virtue of fast gas/liquid mass transfer and short and precise residence time control in flow microreactors. We anticipate that this new tactic will provide an access to a wide range of carboxylic acids and their derivatives bearing electrophilic functional groups. Further work is in progress to explore a wide range of applications of the present method.

\section{Acknowledgements}

This work was partially supported by the Grant-in-Aid for Scientific Research on Innovative Areas "Reaction Integration (No. 2105)". We also thank Taiyo Nippon Sanso for providing a low temperature cooling device and partial financial support and ARKRAY, Inc. for technical support for the reaction of SFB with the cyclic peptide.

\section{References}

[1] a) P. Braunstein, D. Matt, D. Nobel, Chem. Rev. 1988, 88, 747; b) T. Sakakura, J. C. Choi, H. Yasuda, Chem. Rev. 2007, 107, 2365; c) I. Omae, Coord. Chem. Rev. 2012, 256, 1384.

[2] M. Aresta, A. Dibenedetto, Dalton Trans. 2007, 2975.

[3] a) J. Louie, J. E. Gibby, M. V. Farnworth, T. N. Tekavec, J. Am. Chem. Soc. 2002, 124, 15188; b) J. Louie, Curr. Org. Chem. 2005, 9, 605; c) H. Ochiai, M. Jang, K. Hirano, H. Yorimitsu, K. Oshima, Org. Lett. 2008, 10, 2681; d) C. S. Yeung, V. M. Dong, J. Am. Chem. Soc. 2008, 130, 7826; e) A. Correa, R. Martín, Angew. Chem. 2009, 121, 6317; Angew. Chem. Int. Ed. 2009, 48, 6201; f) W. Z. Zhang, W. J. Li, X. Zhang, H. Zhou, X. B. Lu, Org. Lett. 2010, 12, 4748; g) L. Zhang, J. Cheng, T. Ohishi, Z. Hou, Angew. Chem. 2010, 122, 8852; Angew. Chem. Int. Ed. 2010, 49, 8670; h) I. I. F. Boogaerts, S. P. Nolan, J. Am. Chem. Soc. 2010, 132, 8858; i) O. Vechorkin, N. Hirt, X. Hu, Org. Lett. 2010, 12, 3567; j) I. I. F. Boogaerts, G. C. Fortman, M. R. L. Furst, C. S. J. Cazin, S. P. Nolan, Angew. Chem. 2010, 122, 8856; Angew. Chem. Int. Ed. 2010, 49, 8674; k) S. N. Riduan, Y. Zhang, Dalton Trans. 2010, 39, 3347; l) I. I. F. Boogaerts, S. P. Nolan, Chem. Commun. 2011, 47, 3021; m) Y. Zhang, S. N. Riduan, Angew. Chem. 2011, 123, 6334; Angew. Chem. Int. Ed. 2011, 50, 6210; n) S. Li, W. Yuan, S. Ma, Angew. Chem. 123, 2626; Angew. Chem. Int. Ed. 2011, 50, 2578; o) M. Cokoja, C. Bruckmeier, B. Rieger, W. A. Herrmann, F. E. Kühn, Angew. Chem. 2011, 123, 8662; Angew. Chem. Int. Ed. 2011, 50, 8510; p) T. Fujihara, T. Xu, K. Semba, J. Terao, Y. Tsuji, Angew. Chem. Int. Ed. 2011, 50, 523; q) X. Zhang, W. Z. Zhang, X. Ren, L. L. Zhang, X. B. Lu, Org. Lett. 2011, 13, 2402; r) K. Inamoto, N. Asano, K. Kobayashi, M. Yonemoto, Y. Kondo, Org. Biomol. Chem. 2012, 10, 1514; s) T. Fujihara, K. Nogi, T. Xu, J. Terao, Y. Tsuji, J. Am. Chem. Soc. 2012, 134, 9106.

[4] a) I. Mutule, E. Suna, Tetrahedron 2005, 61, 11168; b) D. Y. Tang, A. Lipman, G. J. Meyer, C. N. Wan, A. P. Wolf, J. Labelled Compd. Radiopharm. 1979, 16, 435; c) W. Neugebauer, T. Clark, P. v. R. Schleyer, Chem. Ber. 1983, 116, 3283; d) E. J. Soloski, C. Tamborski, J. Organomet. Chem. 1978, $157,373$.

[5] Some examples of generation and reactions of short-lived organolithiums in flow: a) H. Usutani, Y. Tomida, A. Nagaki, H. Okamoto, T. Nokami, J. Yoshida, J. Am. Chem. Soc. 2007, 129, 3046; b) A. Nagaki, E. Takizawa, J. Yoshida, J. Am. Chem. Soc. 2009, 131, 1654; c) Y. Tomida, A. Nagaki, J. Yoshida, J. Am. Chem. Soc. 2011, 133, 3744; d) H. Kim, A. Nagaki, J. Yoshida, Nat. Commun. 2011, 2, 264; e) A. Nagaki, C. Matsuo, S. Kim, K. Saito, A. Miyazaki, J. Yoshida, Angew. Chem. Int. Ed. 2012, 51, 3245; f) A. Nagaki, D. Yamada, S. Yamada, M. Doi, D. Ichinari, Y. Tomida, N. Takabayashi, J. Yoshida, Aust. J. Chem. 2013, 66, 199.

[6] Books on flow microreactor synthesis: a) W. Ehrfeld, V. Hessel, H. Löwe, Microreactors; Wiley-VCH: Weinheim, 2000; b) V. Hessel, S. Hardt, H. Löwe, Chemical Micro Process Engineering; Wiely-VCH Verlag: Weinheim, 2004; c) J. Yoshida, Flash Chemistry. Fast Organic Synthesis in Microsystems: Wiley-Blackwell, 2008; d) V. Hessel, A. Renken, J. C. Schouten, J. Yoshida, Micro Precess Engineering: Wiley-Blackwell, 2009.

[7] Reviews on flow microreactor synthesis: a) K. Jähnisch, V. Hessel, H. Löwe, M. Baerns, Angew. Chem. Int. Ed. 2004, 43, 406; b) G. N. Doku, W. Verboom, D. N. Reinhoudt, A. van den Berg, Tetrahedron 2005, 61, 2733; c) J. Yoshida, A. Nagaki, T. Iwasaki, S. Suga, Chem. Eng. Tech. 2005, 3, 259; d) P. Watts, S. J. Haswell, Chem. Soc. Rev. 2005, 34, 235; e) K. Geyer, J. D. C. Codée, P. H. Seeberger, Chem. Eur. J. 2006, 12, 8434; f) A. J. 
deMello, Nature. 2006, 442, 394; g) H. Song, D. L. Chen, R. F. Ismagilov, Angew. Chem. Int. Ed. 2006, 45, 7336; h) J. Kobayashi, Y. Mori, S. Kobayashi, Chem. Asian. J. 2006, 1, 22; i) M. Brivio, W. Verboom, D. N. Reinhoudt, Lab Chip. 2006, 6, 329; j) B. P. Mason, K. E. Price, J. L. Steinbacher, A. R. Bogdan, D. T. McQuade, Chem. Rev. 2007, 107, 2300; k) B. Ahmed-Omer, J. C. Brandt, T. Wirth, Org. Biomol. Chem. 2007, 5, 733; l) P. Watts, C. Wiles, Chem. Commun. 2007, 443; m) T. Fukuyama, M. T. Rahman, M. Sato, I. Ryu, Synlett 2008, 151; n) J. Yoshida, A. Nagaki, T. Yamada, Chem. Eur. J. 2008, 14, 7450; o) R. L. Hartman, K. F. Jensen, Lab Chip, 2009, 9, 2495; p) J. P. McMullen, K. F. Jensen, Annu. Rev. Anal. Chem. 2010, 3, 19; q) J. Yoshida, H. Kim, A. Nagaki, ChemSusChem 2011, 4, 331; r) C. Wiles, P. Watts, Green Chem. 2012, 14, 38; s) A. Kirschining, L. Kupracz, J. Hartwig, Chem. Lett. 2012, 41, 562; t) J. Yoshida, Y. Takahashi, A. Nagaki, Chem. Commun. 2013, 49, 9896; u) D. T. McQuade, P. H. Seeberger, J. Org. Chem. 2013, 78, 6384. v) K. S. Elvira, X. C. i Solvas, R. C. R. Wootton, A. J. deMello, Nat. Chem. 2013, 5, 905; w) J. C. Pastre, D. L. Browne, S. V. Ley, Chem. Soc. Rev. 2013, 42, 8849; x) I. R. Baxendale, J. Chem. Technol. Biotechnol. 2013, 88, 519.

[8] Some selected recent examples: a) D. Cantillo, M. Baghbanzadeh, C. O. Kappe, Angew. Chem. Int. Ed. 2012, 51, 10190; b) W. Shu, S. L. Buchwald, Angew. Chem. Int. Ed. 2012, 51, 5355; c) A. Nagaki, Y. Moriwaki, J. Yoshida, Chem. Commun. 2012, 48, 11211; d) F. Lévesque, P. H. Seeberger, Angew. Chem. Int. Ed. 2012, 51, 1706; e) K. C. Basavaraju, S. Sharma, R. A. Maurya, D.-P. Kim, Angew. Chem. Int. Ed. 2013, 52, 6735; f) C. Brancour, T. Fukuyama, Y. Mukai, T. Skrydstrup, I. Ryu, Org. Lett. 2013, 15, 2794; g) J. D. Nguyen, B. Reiß, C. Dai, C. R. J. Stephenson, Chem. Commun. 2013, 49, 4352; h) C. Battilocchio, J. M. Hawkins, S. V. Ley, Org. Lett. 2013, 15, 2278; i) A. S. Kleinke, T. F. Jamison, Org. Lett. 2013, 15, 710; j) K. Asano, Y. Uesugi, J. Yoshida, Org. Lett. 2013, 15, 2398; k) A. Nagaki, D. Ichinari, J. Yoshida, Chem. Commun. 2013, 49, 3242; l) L. Guetzoyan, N. Nikbin, I. R. Baxendale, S. V. Ley, Chem. Sci. 2013, 4, 764; m) S. Fuse, Y. Mifune, T. Takahashi, Angew. Chem. Int. Ed. $2014,53,851$.

[9] a) A. Günther, S. A. Khan, M. Thalmann, F. Trachsel, K. F. Jensen, Lab Chip 2004, 4, 278; b) J. Kobayashi, Y. Mori, K. Okamoto, R. Akiyama, M. Ueno, T. Kitamori, S. Kobayashi, Science 2004, 304, 1305; c) M. T. Rahman, T. Fukuyama, N. Kamata, M. Sato, I. Ryu, Chem. Commun. 2006, 2236; d) T. Fukuyama, M. T. Rahman, N. Kamata, I. Ryu, Beilstein J. Org. Chem. 2009, 5, No. 34; e) N. de Mas, A. Günther, M. A. Schmidt, K. F. Jensen, Ind. Eng. Chem. Res. 2009, 48, 1428; f) N. Wang, T. Matsumoto, M. Ueno, H. Miyamura, S. Kobayashi, Angew. Chem. Int. Ed. 2009, 48, 4744; g) O. Trapp, S. K. Weber, S. Bauch, W. Hofstadt, Angew. Chem. Int. Ed. 2007, 46, 7307; h) M. N. Kashid, L. Kiwi-Minsker, Ind. Eng. Chem. Res. 2009, 48, 6465; i) R. V. Jones, L. Godorhazy, N. Varga, D. Szalay, L. Urge, F. Darvas, J. Comb. Chem. 2006, 8, 110; j) W. Li, K. Liu, R. Simms, J. Greener, D. Jagadeesan, S. Pinto, A. Günther, E. Kumacheva, J. Am. Chem. Soc. 2012, 134, 3127; k) B. Gutmann, P. Elsner, D. Roberge, C. O. Kappe, ACS Catalysis, 2013, 3, 2669; l) F. Mastronardi, B. Gutmann, C. O. Kappe, Org. Lett., 2013, 15, 5590; m) B. Pieber, S. T. Martinez, D. Cantillo, C. O. Kappe, Angew. Chem. Int. Ed., 2013, 52, 10241; n) B. Pieber, C. O. Kappe, Green Chem., 2013, 15, 320.

[10] Carboxylation in flow systems: a) A. Polyzos, M. O'Brien, T. P. Petersen, I. R. Baxendale, S. V. Ley, Angew. Chem. Int. Ed. 2011, 50, 1190; b) J. A. Kozak, J. Wu, X. Su, F. Simeon, T. A. Hatton, T. F. Jamison, J. Am. Chem. Soc. 2013, 135, 18497; c) L. Kupracz, A. Kirschning, Adv. Synth. Catal. 2013, 355, 3375.

[11] A. Nagaki, H. Kim, J. Yoshida, Angew. Chem. Int. Ed. 2009, 48, 8063.

[12] A. Nagaki, H. Kim, C. Matuo, J. Yoshida, Org. Biomol. Chem. 2010, 8, 1212.

[13] A. Nagaki, H. Kim, J. Yoshida, Angew. Chem. Int. Ed. 2008, 47, 7833.

[14] The use of one equivalent of carbon dioxide resulted in lower yield of the product (70\%).

[15] a) S. M. Ametamey, M. Honer, P. A. Schubiger, Chem. Rev. 2008, 108, 1501; b) M. Tredwell, V. Gouverneur, Angew. Chem. Int. Ed. 2012, 51, 11426 and references therein.

[16] B. H. Rotstein, S. H. Liang, J. P. Holland, T. L. Collier, J. M. Hooker, A. A. Wilson, N. Vasdev, Chem. Commun., 2013, 49, 5621 and references therein.

Received: ((will be filled in by the editorial staff))

Revised: ((will be filled in by the editorial staff))

Published online: ((will be filled in by the editorial staff)) 


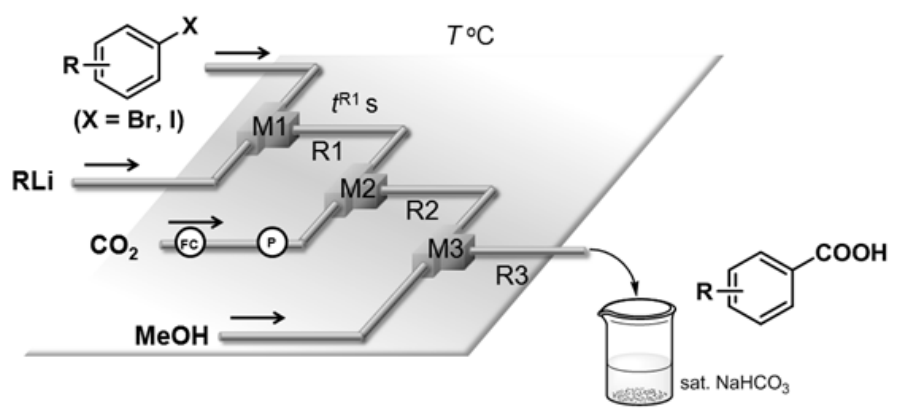

Figure 1. A flow microreactor system for the reactions of organolithiums with $\mathrm{CO}_{2}$ gas. T-shaped micromixers: $\mathrm{M} 1, \mathrm{M} 2, \mathrm{M} 3$; microtube reactors: R1, R2, R3; flow controller: FC; pressure gage: P.

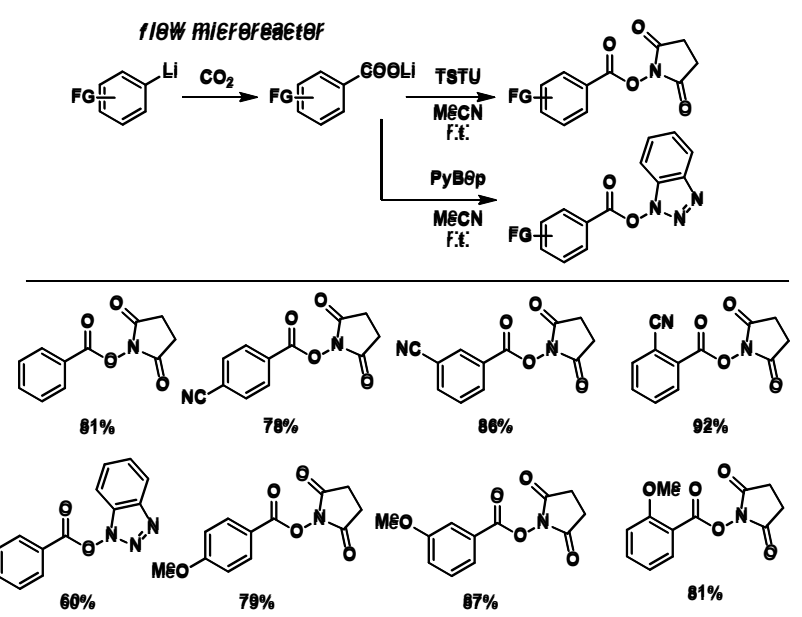

Figure 2. Flow microreactor synthesis of activative esters.

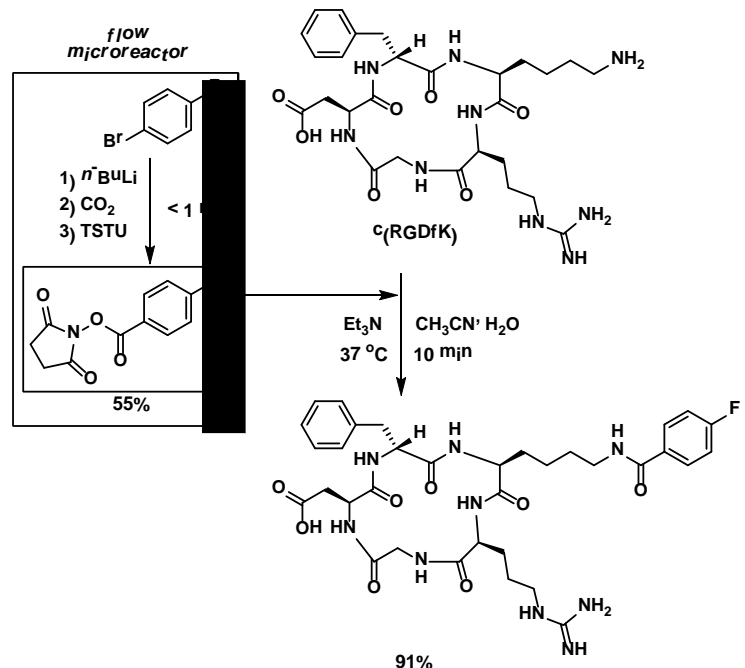

Figure 3. Coupling reaction of $c y c l o(A r g-G l y-A s p-D-P h e-L y s)$ (c(RGDfK)) with N-succinimidyl-4-fluorobenzoate (SFB).

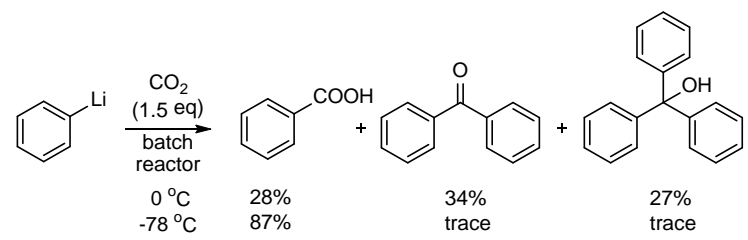

Scheme 1. Reaction of phenyllithium with carbon dioxide using a conventional macro batch reactor. 
Table 1. Carboxylation of aryllithiums with $\mathrm{CO}_{2}$ gas using a flow microreactor system. ${ }^{a}$

\begin{tabular}{|c|c|c|c|c|c|}
\hline Halobenzene & RLi & $\begin{array}{c}T \\
\left({ }^{\circ} \mathrm{C}\right)\end{array}$ & $t^{\mathrm{R} 1}(\mathrm{~s})$ & product & $\begin{array}{c}\text { yield } \\
(\%)\end{array}$ \\
\hline$>1$ & PhLi & -20 & 0.014 & & 78 \\
\hline & PhLi & -20 & 0.014 & & 85 \\
\hline & PhLi & -20 & 0.014 & & 59 \\
\hline & BuLi & -20 & 0.33 & & 75 \\
\hline & BuLi & -20 & 0.33 & & 88 \\
\hline & BuLi & -20 & 0.33 & & 87 \\
\hline & PhLi & -60 & 0.055 & O & 87 \\
\hline & PhLi & -60 & 0.014 & & 77 \\
\hline & PhLi & -60 & 0.014 & & 89 \\
\hline & BuLi & -20 & 2.4 & & 86 \\
\hline & BuLi & -20 & 2.4 & & 88 \\
\hline & BuLi & -20 & 2.4 & & 87 \\
\hline & BuLi & -20 & 4.7 & & 85 \\
\hline & & 0 & 4.7 & & 87 \\
\hline
\end{tabular}

a The reactions were carried out using 1.05 equiv of a lithiating agent and 1.50 equiv of $\mathrm{CO}_{2}$. 


\section{Microreactors}

Aiichiro Nagaki, Yusuke Takahashi, and Jun-ichi Yoshida*

Page - Page

\section{Extremely Fast Gas/Liquid}

Reactions in Flow Microreactors:

Carboxylation of Short-Lived

Organolithiums

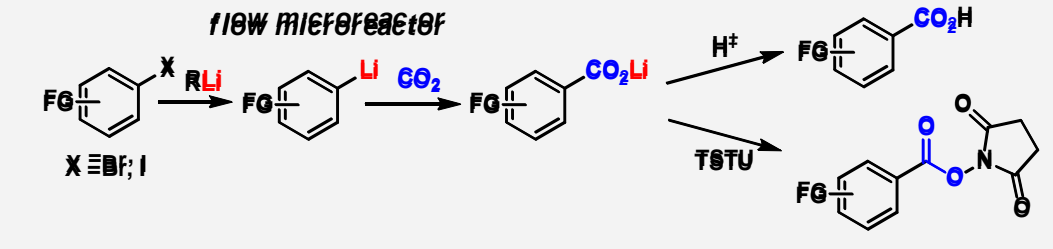

Carboxylation of short-lived in a flow microreactor system. The organolithiums bearing electrophilic successful reactions indicates that functional groups such as nitro, gas/liquid mass transfer and the cyano, and alkoxycarbonyl groups subsequent chemical reaction with with $\mathrm{CO}_{2}$ to give carboxylic acids $\mathrm{CO}_{2}$ are extremely fast. and active esters was accomplished

Keywords: microreactors · flow chemistry · carboxylation · lithiation 